\title{
Imprinting substrate structures onto a nematic liquid crystal
}

Cite as: J. Chem. Phys. 137, 144703 (2012); https://doi.org/10.1063/1.4757391

Submitted: 06 August 2012 - Accepted: 19 September 2012 • Published Online: 11 October 2012

Manuel Greschek, Keith E. Gubbins and Martin Schoen

ARTICLES YOU MAY BE INTERESTED IN

Defect topologies in a nematic liquid crystal near a patchy colloid

The Journal of Chemical Physics 136, 194703 (2012); https://doi.org/10.1063/1.4717619

Mean-field density functional theory of a nanoconfined classical, three-dimensional

Heisenberg fluid. I. The role of molecular anchoring

The Journal of Chemical Physics 144, 194704 (2016); https://doi.org/10.1063/1.4949330

A novel model for smectic liquid crystals: Elastic anisotropy and response to a steady-state flow

The Journal of Chemical Physics 145, 164903 (2016); https://doi.org/10.1063/1.4965711

The Journal

SPECIAL TOPIC: Low-Dimensional

of Chemical Physics Materials for Quantum Information Science 


\title{
Imprinting substrate structures onto a nematic liquid crystal
}

\author{
Manuel Greschek, ${ }^{1}$ Keith E. Gubbins, ${ }^{2}$ and Martin Schoen ${ }^{1,2}$ \\ ${ }^{1}$ Stranski-Laboratorium für Physikalische und Theoretische Chemie, Fakultät für Mathematik und \\ Naturwissenschaften, Technische Universität Berlin, Straße des 17. Juni 135, 10623 Berlin, Germany \\ ${ }^{2}$ Department of Chemical and Biomolecular Engineering, Engineering Building I, Box 7905, \\ North Carolina State University, 911 Partners Way, Raleigh, North Carolina 27695, USA
}

(Received 6 August 2012; accepted 19 September 2012; published online 11 October 2012)

\begin{abstract}
By means of Monte Carlo simulations in the grand canonical ensemble we study the morphology of the nematic phase of a simple model liquid crystal interacting with an alternating sequence of chemically different stripes. The stripes anchor molecules such that their orientation is either parallel or perpendicular with the substrate plane. The different molecular orientations are realized through anchoring functions that cause an energetic penalty for molecules oriented in an undesired fashion. We consider combinations of monostable and degenerate anchoring fields. The nature of the nematic phase is characterized through both the local nematic order parameter and the associated local director field. We observe states of uniaxial or biaxial symmetry depending on the ratio of stripe widths and the range of fluid-substrate attraction. In some cases the specific substrate pattern causes regions of biaxial symmetry to coexist with a bulk-like regime sufficiently far away from the substrates in which the local director field indicates a (homogeneous) bent state of the nematic liquid crystal. (C) 2012 American Institute of Physics. [http://dx.doi.org/10.1063/1.4757391]
\end{abstract}

\section{INTRODUCTION}

An important feature of bulk nematic liquid crystals is their birefringence which makes this class of materials particularly useful with regard to displays. ${ }^{1}$ In the realm of optical switches - to name another important example-droplets of nematic liquid crystals have been suggested as microresonators with a wide range of applications. ${ }^{2}$ For the nematic phase to be birefringent a spatially homogeneous director field is the fundamental requirement. However, if the nematic liquid crystal interacts with a solid substrate the director field is perturbed and may become spatially inhomogeneous over large distances such that the material is no longer entirely birefringent but exhibits defects that can be detected and analyzed optically because the correlation length associated with the perturbation is in the range of the wavelength of visible light. This has been demonstrated by Guzmán et al. who therefore propose nematic liquid crystals in confinement as biosensors. $^{3}$

If the solid substrate is endowed with some sort of substructure which may be topological or chemical in nature, ${ }^{4}$ even more complex perturbations of the local director field are to be expected that may have important repercussions in the realm of nanooptical devices. ${ }^{5,6}$ Experimentally, it is nowadays feasible to prepare submicro-textured substrates in a variety of ways ${ }^{7-9}$ including microcontact printing ${ }^{10}$ or nanorubbing using the tip of an atomic force microscope. ${ }^{11}$ In this latter work the authors succeeded in setting up a solid substrate with a checkerboard pattern where the liquid crystal molecules are anchored planar but with orthogonal orientations between neighboring square unit domains. Individual domains can be made as small as $0.5 \mu \mathrm{m} \times 0.5 \mu \mathrm{m}$ and are therefore roughly comparable in size to the nanopatterns studied in this work. However, with more sophisticated patterning techniques it seems conceivable that the pattern size studied in Ref. 11 is by no means the lower limit of what can be realized experimentally. The two diagonals of the square domains in Ref. 11 are therefore equally stable with respect to the macroscopic alignment of the liquid crystal. By means of an electric field it is possible to switch between these two equivalent directions. This opens the possibility to use nanostructured domains as microscopic switches.

On the theoretical side Kondrat and Poniewierski investigated the anchoring of a liquid crystal at a solid substrate patterned with alternating stripes at which molecules align themselves in a parallel and in a homeotropic (normal to the substrate plane) fashion, respectively. ${ }^{12}$ By minimizing the free energy of their system the authors investigate the impact of the relative width of the differently anchoring stripes on the phase behavior of the liquid crystal. Later Poniewierski and Kondrat studied a system in which a liquid crystal is sandwiched between two planar substrates decorated with alternating planar and homeotropically anchoring stripes. ${ }^{13}$ However, in this work the stripes on the two opposite substrates are shifted with respect to one another and the distortion free energy of the liquid-crystalline phase is investigated as a function of substrate separation. The combined impact of substrate topology and chemical decoration has been studied by Harnau et al. ${ }^{14}$

Within the framework of Frank-Oseen theory Kondrat et al. computed the solvation force for a liquid crystal interacting with a patterned substrate. ${ }^{15}$ Haddadan and Dietrich studied lateral and normal forces in a liquid crystal confined between plane parallel substrates decorated with periodically varying regions of different anchoring conditions. ${ }^{16}$

Atherton and Sambles investigated the director field in a nematic liquid crystal in a twisted nematic cell set up with micropatterned substrates. ${ }^{17}$ They could show that the 
director field is very sensitive to small changes in the elastic constants characterizing the liquid crystal. The phase diagram of a liquid crystal interacting with a micropatterned substrate has been calculated by Atherton who observed four distinctly different topologies of the local director field depending on various model parameters. ${ }^{18}$

These earlier studies usually take an ansatz for the free energy which is subsequently minimized to obtain information about properties characterizing the thermodynamically stable phase. Thus, only limited insight into molecular causes of substrate-induced formation of orientation patterns can be provided by these approaches. It therefore seems worthwhile to complement the already existing, interesting theoretical studies by molecular simulation. In this work we present Monte Carlo (MC) simulations of a simple liquid crystal confined between chemically striped walls where the stripes are distinguished by different anchoring scenarios that lead to the formation of domains. In these domains liquidcrystal molecules (i.e., mesogens) are oriented either in a homeotropic or in a planar fashion with respect to the substrate plane. The differently oriented domains are separated by interfaces that are isotropic with respect to the distribution of molecular orientations.

The remainder of our paper is organized as follows. In Secs. II and III we introduce our model system and present technical details of our calculations. Results for various anchoring conditions and model parameters are presented in Sec. IV. They are summarized and discussed in the concluding Sec. V.

\section{MODEL}

We consider a system of $N$ mesogens interacting with each other in a pairwise additive fashion such that the total fluid-fluid (ff) configurational potential energy may be cast as

$$
\Phi_{\mathrm{ff}}(\boldsymbol{R}, \widehat{\boldsymbol{U}})=\frac{1}{2} \sum_{i=1}^{N} \sum_{\substack{j=1 \\ j \neq i}}^{N} \varphi\left(\boldsymbol{r}_{i j}, \widehat{\boldsymbol{u}}_{i}, \widehat{\boldsymbol{u}}_{j}\right),
$$

where $\boldsymbol{r}_{i j} \equiv \boldsymbol{r}_{i}-\boldsymbol{r}_{j}$ is the distance vector between the centers-of-mass of mesogens $i$ and $j$. In Eq. (2.1), $\boldsymbol{R} \equiv\left\{\boldsymbol{r}_{1}, \boldsymbol{r}_{2}, \ldots, \boldsymbol{r}_{N}\right\}$ and $\widehat{\boldsymbol{U}} \equiv\left\{\widehat{\boldsymbol{u}}_{1}, \widehat{\boldsymbol{u}}_{1}, \ldots, \widehat{\boldsymbol{u}}_{N}\right\}$ are shorthand notations for the sets of center-of-mass coordinates and unit vectors specifying the orientation of the mesogens, respectively. Throughout this paper "..." denotes a unit vector.

As in our previous work $^{19-22}$ we decompose the intermolecular interaction potential into an isotropic and an anisotropic contribution,

$$
\varphi\left(\boldsymbol{r}_{i j}, \widehat{\boldsymbol{u}}_{i}, \widehat{\boldsymbol{u}}_{j}\right)=\varphi_{\mathrm{iso}}\left(r_{i j}\right)+\varphi_{\mathrm{anis}}\left(\boldsymbol{r}_{i j}, \widehat{\boldsymbol{u}}_{i}, \widehat{\boldsymbol{u}}_{j}\right),
$$

where $r_{i j}=\left|\boldsymbol{r}_{i j}\right|$ and the isotropic contribution is given by the well-known Lennard-Jones potential:

$$
\varphi_{\mathrm{iso}}\left(r_{i j}\right)=4 \varepsilon_{\mathrm{ff}}\left[\left(\frac{\sigma}{r_{i j}}\right)^{12}-\left(\frac{\sigma}{r_{i j}}\right)^{6}\right] .
$$

In Eq. (2.3), $\sigma$ is the diameter of a spherically symmetric reference particle and $\varepsilon_{\mathrm{ff}}$ is the depth of the attractive well of $\varphi_{\text {iso }}$. The anisotropic contribution is given by

$$
\varphi_{\text {anis }}\left(\boldsymbol{r}_{i j}, \widehat{\boldsymbol{u}}_{i}, \widehat{\boldsymbol{u}}_{j}\right)=-4 \varepsilon_{\mathrm{ff}}\left(\frac{\sigma}{r_{i j}}\right)^{6} \Psi\left(\widehat{\boldsymbol{r}}_{i j}, \widehat{\boldsymbol{u}}_{i}, \widehat{\boldsymbol{u}}_{j}\right),
$$

where $\widehat{\boldsymbol{r}}_{i j}=\boldsymbol{r}_{i j} / r_{i j}$. In Eq. (2.4), the function $\Psi\left(\widehat{\boldsymbol{r}}_{i j}, \widehat{\boldsymbol{u}}_{i}, \widehat{\boldsymbol{u}}_{j}\right)$ describes the orientation dependence of the intermolecular interactions. It has to be constructed such that $u_{\text {anis }}$ meets certain symmetry requirements. ${ }^{23}$ For instance, the head-tail symmetry of the mesogens has to be preserved, that is $\varphi_{\text {anis }}$ should be invariant if $\widehat{\boldsymbol{u}}_{i}$ and/or $\widehat{\boldsymbol{u}}_{j}$ change sign. Moreover,

$$
\iint \mathrm{d} \widehat{\boldsymbol{u}}_{i} \mathrm{~d} \widehat{\boldsymbol{u}}_{j} \varphi_{\text {anis }}\left(\boldsymbol{r}_{i j}, \widehat{\boldsymbol{u}}_{i}, \widehat{\boldsymbol{u}}_{j}\right)=0 .
$$

These requirements are met if

$$
\begin{aligned}
\Psi\left(\widehat{\boldsymbol{r}}_{i j}, \widehat{\boldsymbol{u}}_{i}, \widehat{\boldsymbol{u}}_{j}\right)= & 5 \varepsilon_{1} P_{2}\left(\widehat{\boldsymbol{u}}_{i} \cdot \widehat{\boldsymbol{u}}_{i}\right) \\
& +5 \varepsilon_{2}\left[P_{2}\left(\widehat{\boldsymbol{u}}_{i} \cdot \widehat{\boldsymbol{r}}_{i j}\right)+P_{2}\left(\widehat{\boldsymbol{u}}_{j} \cdot \widehat{\boldsymbol{r}}_{i j}\right)\right],
\end{aligned}
$$

which is obtained from a summation of certain Wigner matrices, ${ }^{23}$ where

$$
P_{2}(x) \equiv \frac{1}{2}\left(3 x^{2}-1\right)
$$

is the second Legendre polynomial and $\varepsilon_{1}=0.04$ and $\varepsilon_{2}=-0.08$ are chosen throughout this work.

The model potential introduced in Eqs. (2.1)-(2.7) describes the interaction between a pair of thermotropic mesogens. In our model liquid-crystalline phases come about because of the anisotropic attraction and not because of a large, anisometric excluded volume (i.e., a large aspect ratio) as in the more conventional Gay-Berne and Kihara models of liquid crystals or their various hybrids.

The liquid crystal is confined between two planar, chemically structured solid substrates separated by a distance $s_{\mathrm{Z}}$ along the $z$-axis of a space-fixed Cartesian coordinate system. The setup of the model is illustrated by the cartoon presented in Fig. 1. Hence, a fluid-substrate (fs) contribution to the total configurational potential energy arises which may be cast as

$$
\Phi_{\mathrm{fs}}(X, Z, \widehat{\boldsymbol{U}})=\sum_{k=1}^{2} \sum_{i=1}^{N} \phi^{(k)}\left(x_{i}, z_{i}, \widehat{\boldsymbol{u}}_{i} ; \kappa\right),
$$

where $X \equiv\left\{x_{1}, x_{2}, \ldots, x_{N}\right\}$ and $Z \equiv\left\{z_{1}, z_{2}, \ldots, z_{N}\right\}$ are shorthand notations. In Eq. (2.8)

$$
\begin{aligned}
\phi^{(k)}\left(x_{i}, z_{i}, \widehat{\boldsymbol{u}}_{i} ; \kappa\right)= & \varepsilon_{\mathrm{fs}}\left[a_{1}\left(\frac{\sigma}{z_{i} \pm s_{\mathrm{Z}} / 2}\right)^{10}\right. \\
& \left.-a_{2} \frac{\exp \left(-\kappa\left|z_{i} \pm s_{\mathrm{Z}} / 2\right|\right)}{\left|z_{i} \pm s_{\mathrm{z}} / 2\right|} g\left(x_{i}, \widehat{\boldsymbol{u}}_{i}\right)\right],
\end{aligned}
$$

where $g\left(x_{i}, \widehat{\boldsymbol{u}}_{i}\right)$ is the so-called anchoring function (see below). We assume the lower $(k=1)$ substrate to be located at $z=-s_{\mathrm{Z}} / 2$ whereas the upper one $(k=2)$ is located at $z=+s_{z} / 2$. Hence, the fluid-substrate potential function consists of a repulsive part and an attractive, Yukawa part where $\kappa^{-1}$ is a screening length such that the range of $\phi^{(k)}$ of attractive fluid-substrate interactions becomes shorter with increas- 


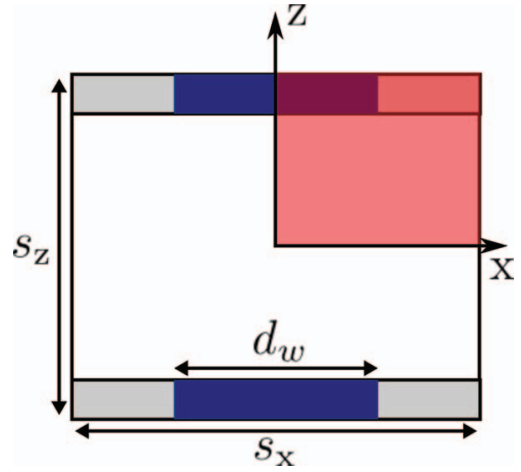

FIG. 1. Cartoon of the setup of the simulation cell in the $x-z$ plane. Substrates are separated by a distance $s_{\mathrm{z}}$; the width of the simulation cell along the $x$-axis is $s_{\mathrm{X}}$. Periodic boundary conditions are applied at $x= \pm s_{\mathrm{X}} / 2$ and $y= \pm s_{\mathrm{y}} / 2$ (normal to the paper plane). Substrates consist of a central stripe of width $d_{\mathrm{w}}$ shaded in dark blue where mesogens are anchored either directionally or parallel; at the two outer stripes of width $\left(s_{\mathrm{X}}-d_{\mathrm{w}}\right) / 2$ shaded in gray the anchoring is always homeotropic. System properties are translationally invariant along the $y$-axis; in the $x-z$ plane mirror symmetry exists with respect to both the $x$ and $z$ axes. Local properties are therefore displayed only in the first quadrant shaded in red.

ing $\kappa$. A very scholarly discussion of the Yukawa potential and the disparate physical situations to which it applies was given by Rowlinson. ${ }^{24}$ Here we use the Yukawa attraction out of mere convenience because the range of the attractive contribution to $\phi^{(k)}$ can be changed continuously by varying $\kappa .^{22}$ To fix the depth of the attractive well and the location of the minimum of $\phi^{(k)}$ we introduced the dimensionless parameters

$$
\begin{gathered}
a_{1}=-\frac{\kappa \sigma+1}{\kappa \sigma-9}, \\
a_{2}=-\frac{10 \exp (\kappa \sigma)}{\kappa \sigma-9}
\end{gathered}
$$

in Eq. (2.9). These parameters guarantee that

$$
\begin{gathered}
\left.\frac{\mathrm{d} \phi^{(k)}}{\mathrm{d} z}\right|_{z=z_{\min }}=0, \\
\left.\phi^{(k)}\right|_{z=z_{\min }}=-\varepsilon_{\mathrm{fs}},
\end{gathered}
$$

where $z_{\min } \equiv \pm s_{\mathrm{z}} / 2 \mp \sigma$ denotes the location of the minimum of $\phi^{(k)}$ relative to the upper and lower substrate, respectively. We illustrate the form of the fluid-substrate interaction potential for three values of $\kappa$ by the plots in Fig. 2.

Because $\phi^{(k)}\left(x_{i}, z_{i}, \widehat{\boldsymbol{u}}_{i} ; \kappa\right)$ in Eq. (2.9) does not depend on $y_{i}$ local system properties are translationally invariant in the $y$-direction. Moreover, assuming the origin of the coordinate system to be located at the center of the simulation cell all local properties are symmetric with respect to both the $x$ and $z$ axes. This permits us to average our data over corresponding points in all four quadrants. The result of this averaging then needs to be represented only for points in the upper right quadrant shaded in red in Fig. 1.

It should be noted at this point that values for $\kappa$ have been chosen here with no particular experimental setup in mind. By varying $\kappa$ we merely intend to see what impact a variation of the interaction range normal to the substrate plane has on defect topologies emerging in the adjacent nematic liquid crys-

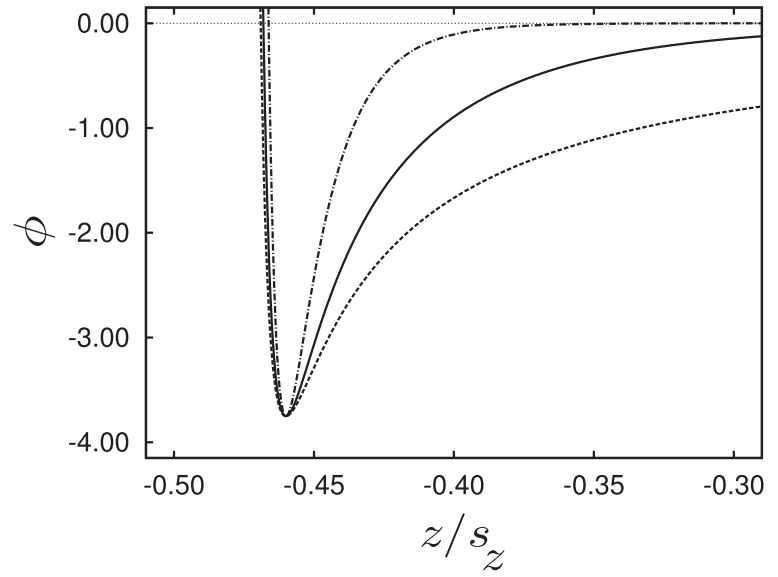

FIG. 2. Fluid-substrate potential $\phi^{(1)}$ at lower substrate $\left(z_{\text {wall }}=-s_{\mathrm{z}} / 2\right)$ as a function of position $z$. Curves have been generated for $\kappa \sigma=0.0(----)$, $\kappa \sigma=0.45(-), \kappa \sigma=2.00(-\cdot-\cdot), \varepsilon_{\mathrm{fs}} / \varepsilon_{\mathrm{ff}}=15 / 4$, and for $g\left(x_{i}, \widehat{\boldsymbol{u}}_{i}\right)=1$ [see Eq. (2.9)]. The value of $\varepsilon_{\mathrm{fs}}$ is used throughout this work.

tal. Experimentally this might be realized through differently shielded, additional external (e.g., electric or magnetic) fields.

The function $g\left(x_{i}, \widehat{\boldsymbol{u}}_{i}\right)$ in Eq. (2.9) is the anchoring function which serves to discriminate energetically different orientations of mesogens at different parts of the substrate. Throughout this work we shall assume that the substrates of length $s_{\mathrm{X}}$ in the $x$-direction are decorated with a central stripe of width $d_{\mathrm{w}}$ at which the anchoring of a mesogen differs from that at the outer two stripes both of widths $\left(s_{\mathrm{x}}-d_{\mathrm{w}}\right) / 2$. At the outer stripes mesogens are always anchored homeotropically whereas they may be anchored either planar or directionally at the central stripe (see Fig. 1). We realize regions of different anchoring scenarios through the anchoring function [see Eq. (2.9)],

$$
g\left(x_{i}, \widehat{\boldsymbol{u}}_{i}\right) \equiv g_{\uparrow}\left(\widehat{\boldsymbol{u}}_{i}\right)+\left[g_{\leftrightarrow}\left(\widehat{\boldsymbol{u}}_{i}\right)-g_{\uparrow}\left(\widehat{\boldsymbol{u}}_{i}\right)\right] s\left(x_{i} ; d_{\mathrm{w}}\right),
$$

where

$$
s\left(x_{i} ; d_{\mathrm{w}}\right)=\frac{1}{2}\left[\tanh \left(x_{i}-d_{\mathrm{w}}\right)-\tanh \left(x_{i}+d_{\mathrm{w}}\right)\right]
$$

is a switching function varying between 0 and 1 (see Fig. 3). The functions $g_{\leftrightarrow}$ and $g_{\downarrow}$ are specified in Table I.

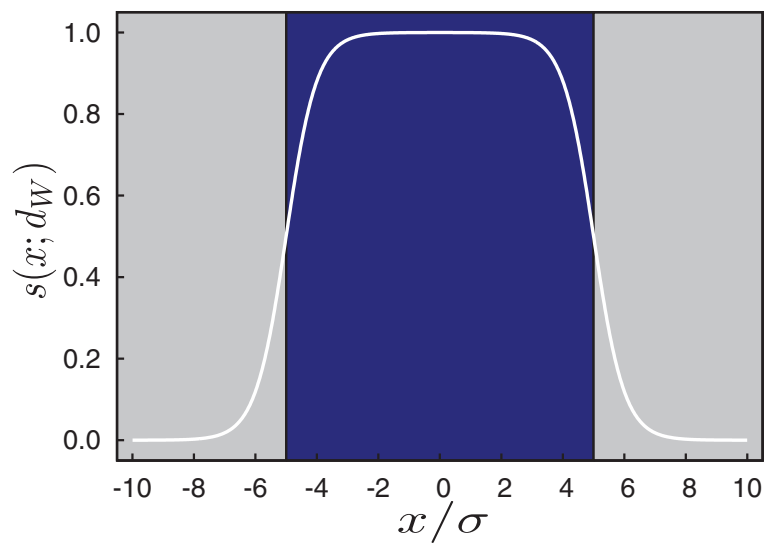

FIG. 3. Switching function $s\left(x ; d_{\mathrm{w}}\right)$ as a function of lateral position for $s_{\mathrm{X}}=20$ and $d_{\mathrm{w}}=10$. In gray shaded areas mesogens are preferentially aligned homeotropically whereas in dark blue shaded areas the alignment is predominantly parallel with the substrate plane. 
TABLE I. Combinations of anchoring functions applied at outer $\left(g_{\uparrow}\right)$ and inner stripes $\left(g_{\leftrightarrow}\right)$, respectively. Acronym hd corresponds to a combination of homeotropic (h) and directional (d) anchoring whereas acronym $\mathrm{hp}$ refers to a combination of homeotropic (h) and planar (p) anchoring. In the terminology of Jerôme ${ }^{25} \mathrm{~h}$ and $\mathrm{d}$ anchoring scenarios are monostable whereas $\mathrm{p}$ anchoring is degenerate.

\begin{tabular}{lcc}
\hline \hline Acronym & $g_{\uparrow}(\widehat{\boldsymbol{u}})$ & $g_{\leftrightarrow}(\widehat{\boldsymbol{u}})$ \\
\hline hd & $\left(\widehat{\boldsymbol{u}} \cdot \widehat{\boldsymbol{e}}_{\mathrm{Z}}\right)^{2}$ & $\left(\widehat{\boldsymbol{u}} \cdot \widehat{\boldsymbol{e}}_{\mathrm{X}}\right)^{2}$ \\
$\mathrm{hp}$ & $\left(\widehat{\boldsymbol{u}} \cdot \widehat{\boldsymbol{e}}_{\mathrm{z}}\right)^{2}$ & $\left(\widehat{\boldsymbol{u}} \cdot \widehat{\boldsymbol{e}}_{\mathrm{X}}\right)^{2}+\left(\widehat{\boldsymbol{u}} \cdot \widehat{\boldsymbol{e}}_{\mathrm{y}}\right)^{2}$ \\
\hline \hline
\end{tabular}

The switching function is defined such that it vanishes in regions where the substrates impose a predominantly homeotropic alignment of a mesogen. In those regions controlled by the central stripe a parallel (planar or directional, see Table I) alignment is preferred because here $s\left(x ; d_{\mathrm{w}}\right) \rightarrow 1$ as $|x| \rightarrow 0$.

\section{TECHNICAL DETAILS}

As in our previous work ${ }^{22}$ we use MC simulations in the grand canonical ensemble employing the algorithm suggested by Gruhn and Schoen ${ }^{26}$ in which the simulation cell is in (virtual) thermodynamic equilibrium with the bulk. Because our system is highly inhomogeneous the use of the grand canonical ensemble in the MC simulations is clearly advantageous. For example, in the grand canonical ensemble we can easily make sure that under equilibrium conditions (i.e., for the same chemical potential $\mu$ and temperature $T$ ) a corresponding bulk system is also in the nematic phase. A similar comparison would be possible only rather indirectly in other suitably defined statistical physical ensembles such as the canonical or an isothermal-isobaric ensemble. Specifying in the former the same mean number density of the bulk and that of the confined system does not imply that the two are in thermodynamic equilibrium because of the inhomogeneity of the confined system. Likewise the (scalar) pressure $P$ bears no obvious relation to elements of the pressure tensor $\mathbf{P}$ of the confined system so that again no conclusions can be drawn about the coexistence between bulk and confined phases.

In the grand canonical ensemble and for the present model fluid microstates are distributed in configuration space according to $\exp \{-\beta[U(\boldsymbol{R}, \widehat{\boldsymbol{U}})-\mu N]-\ln N$ ! $-5 N \ln (\Lambda m / \mathcal{I})\}^{22}$ once the system is equilibrated $(\Lambda$ thermal de Broglie wavelength, $m$ molecular mass, $\mathcal{I}$ moment of inertia). To realize this distribution of microstates the evolution of the system proceeds according to a Markov process consisting of two substeps. In the first of these it is decided with equal probability whether to displace or rotate a sequentially picked mesogen. Whether such an attempted displacement or rotation is accepted is decided upon the associated change in configurational potential energy employing the well-known Metropolis criterion. ${ }^{27}$ The increments by which molecules are displaced or rotated are adjusted during each run such that on average $40 \%-60 \%$ of all attempted displacements or rotations are successful.

Once all $N$ mesogens have been considered in the first step it is decided with equal probability whether to remove an existing mesogen from the system or to create a new one at a randomly chosen position with random orientation. Again, deletion and creation are accepted on the basis of the associated change in configurational potential energy employing a modified Metropolis criterion. ${ }^{27}$ Together the $N$ displacements/rotations and deletion/creation attempts constitute a MC cycle where $N$ is the number of mesogens present at the beginning of a new cycle. To guarantee reliable results, we allow the system to equilibrate for $4 \times 10^{4}$ cycles followed by $1.1 \times 10^{5}$ cycles to compute averages.

To save computer time and because all interaction potentials in our model are short range we cut off the interaction between a pair of mesogens if their center-of-mass distance exceeds the radius $r_{\mathrm{c}}=3.0 \sigma$ of a cutoff sphere centered on one of the two mesogens of the pair. In addition, we employ a combination of a conventional Verlet neighbor list and a linkcell list as described in the book by Allen and Tildesley. ${ }^{28}$ A mesogen is considered a neighbor of a reference mesogen at the center if their center-of-mass distance does not exceed $r_{\mathrm{N}}=3.5 \sigma$. At $r_{\mathrm{c}}$ the interaction potential remains unshifted and no corrections for the neglected long-range interactions are applied to any ensemble average. Moreover, fluidsubstrate interactions are not cut off.

From now on, we express all quantities of interest in dimensionless (i.e., "reduced") units. This means, length is given in units of $\sigma$ and energy in units of $\varepsilon_{\mathrm{ff}}$. All other derived quantities are expressed in suitable combinations of these basic quantities. In all simulations we consider a simulation cell of $s_{\mathrm{x}}=s_{\mathrm{y}}=20, s_{\mathrm{z}}=25$.

For a given value of $\kappa$, we begin by generating a starting configuration at equal stripe widths $d_{\mathrm{w}}=10$ by filling the simulation cell with randomly oriented particles located at random positions. We then let this initially isotropic fluid equilibrate to its nematic state which is the thermodynamically stable equilibrium state given the chosen temperature $T=0.9$ and chemical potential $\mu=-12.089$. For these values the equilibrated fluid is sufficiently deep in the one phase region of the nematic phase indicated by a relatively high value of 0.58 for the nematic order parameter which we shall introduce formally in Sec. III A. ${ }^{22}$ Under these thermodynamic conditions our systems accommodate about 9000 mesogens on average. To study the influence of $d_{\mathrm{w}}$ on the nematic director field we start from the equilibrated nematic configuration at $d_{\mathrm{w}}=10$ and perform a series of simulations where we increase or steadily decrease $d_{\mathrm{w}}$ in small steps between individual MC runs. Subsequent runs are started from the equilibrated configuration of the preceding one.

\section{A. Properties}

Because of the presence of the substrates and their chemical structure the degree of nematic order and the orientation of the mesogens may vary locally. To obtain a quantitative measure of these features of the confined liquid crystal we analyze the local alignment tensor

$$
\mathbf{Q}(\boldsymbol{r}) \equiv \frac{1}{2 \rho(\boldsymbol{r})}\left\langle\sum_{i=1}^{N}\left[3 \widehat{\boldsymbol{u}}_{i} \otimes \widehat{\boldsymbol{u}}_{i}-\mathbf{1}\right] \delta\left(\boldsymbol{r}-\boldsymbol{r}_{i}\right)\right\rangle,
$$


where the operator $\otimes$ represents the tensor (i.e., dyadic) product of the unit vector $\widehat{\boldsymbol{u}}_{i}$ with itself, $\mathbf{1}$ is the unit tensor, and $\delta\left(\boldsymbol{r}-\boldsymbol{r}_{i}\right)$ denotes the Dirac $\delta$-function. In Eq. (3.1)

$$
\rho(\boldsymbol{r})=\left\langle\frac{1}{N} \sum_{i=1}^{N} \delta\left(\boldsymbol{r}-\boldsymbol{r}_{i}\right)\right\rangle
$$

is the local density of the liquid crystal.

Because of the definition in Eq. (3.1), $\mathbf{Q}(\boldsymbol{r})$ is a real, symmetric, traceless, second-rank tensor that may be represented by a $3 \times 3$ matrix. Using Jacobi's method ${ }^{29}$ we compute the three eigenvalues $\lambda_{-}(\boldsymbol{r})<\lambda_{0}(\boldsymbol{r})<\lambda_{+}(\boldsymbol{r})$ of $\mathbf{Q}(\boldsymbol{r})$ numerically. We take $\lambda_{+}(\boldsymbol{r})$ as a measure of local nematic order and drop the subscript + henceforth to ease the notational burden. Moreover, by solving the eigenvalue equation

$$
\mathbf{Q}(\boldsymbol{r}) \widehat{\boldsymbol{n}}(\boldsymbol{r})=\lambda(\boldsymbol{r}) \widehat{\boldsymbol{n}}(\boldsymbol{r}),
$$

we obtain the director field $\widehat{\boldsymbol{n}}(\boldsymbol{r})$ which provides quantitative information about the local orientation of the mesogens in the nematic phase. Because the fluid-substrate potential given in Eq. (2.9) depends only on the position of a mesogen in the $x-z$ plane properties of the liquid crystal are translationally invariant along the $y$-direction. Therefore, we may replace the local nematic order parameter $\lambda(\boldsymbol{r})$ and the director field $\widehat{\boldsymbol{n}}(\boldsymbol{r})$ by $\lambda(x, z)$ and $\widehat{\boldsymbol{n}}(x, z)$, respectively, both of which depend only on position in the $x-z$ plane.

This procedure follows that proposed earlier by Andrienko et al. in their study of topological defects in a liquid crystal. ${ }^{30}$ Notice, however, that the computation of eigenvalues and eigenvectors is based upon an already ensemble-averaged $\mathbf{Q}(x, z)$ whereas conventionally ${ }^{19,31-34}$ the instantaneous alignment tensor is used to compute its eigenvalues which are then ensemble-averaged subsequently. However, for reasons explained elsewhere ${ }^{22}$ this approach could cause a substantial system size dependence of the local nematic order parameter that needs to be corrected for. The present approach avoids that problem by taking the ensemble average first and then diagonalizing $\mathbf{Q}(x, z)$ afterwards. ${ }^{35}$

Another useful measure of local order is provided by the second Legendre polynomial

$$
P_{\alpha}(x, z)=\left\langle\frac{1}{2 N(x, z)} \sum_{i=1}^{N(x, z)}\left[3\left(\widehat{\boldsymbol{u}}_{i}(x, z) \cdot \widehat{\boldsymbol{e}}_{\alpha}\right)^{2}-1\right]\right\rangle,
$$

where $\widehat{\boldsymbol{e}}_{\alpha}$ is a unit vector pointing along the $\alpha$-axis in a Cartesian coordinate system. In analyzing local nematic order via $\mathbf{Q}(x, z)$ or $P_{\alpha}(x, z)$ two main differences should be kept in mind. First, whereas the local nematic director computed from $\mathbf{Q}(x, z)$ is a priori unknown and may in principle point in any direction, the analysis of $P_{\alpha}(x, z)$ explicitly assumes that the local nematic director points along the $\alpha$-axis of the Cartesian coordinate system. If, for example, $\widehat{\boldsymbol{n}}(x, z) \cdot \widehat{\boldsymbol{e}}_{\alpha}=0$, $P_{\alpha}(x, z)<0$ even though $\lambda(x, z)>0$ may reveal a substantial degree of nematic order. Hence, it seems prudent to analyze all three second Legendre polynomials in the $x$-, $y$-, and $z$-directions simultaneously. Second, because of its definition $0 \leq \lambda(x, z) \leq 1$ whereas $-\frac{1}{2} \leq P_{\alpha}(x, z) \leq 1$. Therefore, some caution is required in comparing absolute values of both quantities. (a)

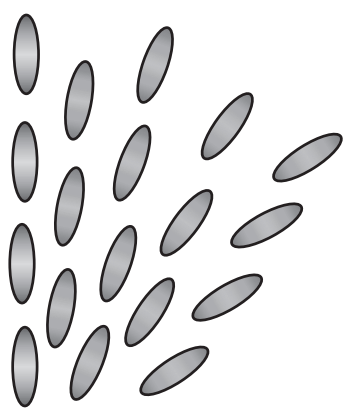

(b)

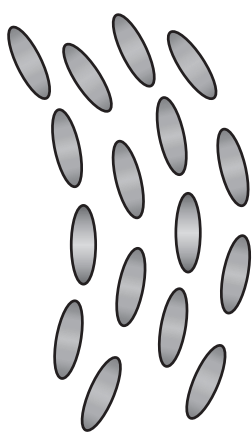

(c)
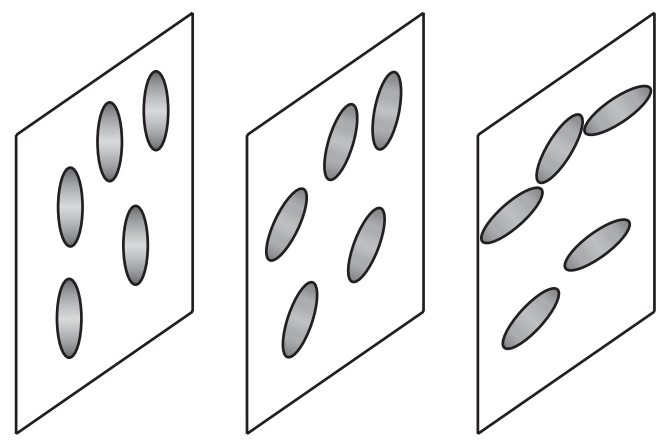

FIG. 4. Schematic representation of local splay (a), bend (b), and twist (c) configurations of a director field.

Another useful quantity to investigate in the context of this work is the so-called Frank free energy density ${ }^{36}$ which in the one-constant approximation may be cast as

$$
\begin{aligned}
\mathcal{F}(\boldsymbol{r})= & \frac{K}{2}\left\{[\nabla \cdot \widehat{\boldsymbol{n}}(\boldsymbol{r})]^{2}+[\widehat{\boldsymbol{n}}(\boldsymbol{r}) \cdot \nabla \times \widehat{\boldsymbol{n}}(\boldsymbol{r})]^{2}\right. \\
& \left.+[\widehat{\boldsymbol{n}}(\boldsymbol{r}) \times \nabla \times \widehat{\boldsymbol{n}}(\boldsymbol{r})]^{2}\right\} .
\end{aligned}
$$

In Eq. (3.5), $K$ is the so-called elastic constant and the three terms on the right side correspond to splay, twist, and bend deformations of the local nematic director field. These three contributions, which are assumed to be the major ones in our case, are depicted schematically in Fig. 4. However, because we have access to the local nematic director field it is not necessary to consider twist, splay, and bend contributions separately. In fact, it proves to be more convenient to use a slightly different form of $\mathcal{F}(\boldsymbol{r})$. This follows by employing the identity $(\nabla \times \boldsymbol{a})^{2}=(\boldsymbol{a} \cdot \nabla \times \boldsymbol{a})^{2}+(\boldsymbol{a} \times \nabla \times \boldsymbol{a})^{2}$ which holds for an arbitrary vector $\boldsymbol{a}$. Thus, we may rewrite Eq. (3.5) more compactly as

$$
\mathcal{F}(\boldsymbol{r})=\frac{K}{2}\left\{[\nabla \cdot \widehat{\boldsymbol{n}}(\boldsymbol{r})]^{2}+[\nabla \times \widehat{\boldsymbol{n}}(\boldsymbol{r})]^{2}\right\} .
$$

Because the local director field is constant in the $y$-direction, the Frank free-energy density also turns out to be a function of position in the $x-z$ plane only. Hence, we may replace the argument $\boldsymbol{r}$ by $x$ and $z$. The Frank free-energy density is directly related to local deformations of the nematic director field. Hence, it may be interpreted as a quantitative measure of long-range elastic forces present in a nematic liquid crystal which are caused by a local perturbation of an otherwise homogeneous director field. Because of its definition it is also important to realize that an analysis of $\mathcal{F}(x, z)$ only makes 
sense in the nematic phase. Moreover, from both Eqs. (3.5) and (3.6) it is immediately obvious that in order to compute $\mathcal{F}(x, z)$ the local nematic director field needs to be differentiated which is done numerically using a standard finitedifference scheme.

However, a small technical problem arises due to the fact that $\widehat{\boldsymbol{n}}(\boldsymbol{r})$ and $-\widehat{\boldsymbol{n}}(\boldsymbol{r})$ are equivalent. This reflects the uniaxial symmetry of our molecules and is a consequence of the mathematical structure of $\mathbf{Q}$ in the eigenvalue Eq. (3.3). Hence, in a straightforward calculation of $\mathcal{F}(x, z)$ by a numerical differentiation of the director field one needs to make sure that neighboring local directors satisfy the condition that their scalar product is non-negative to avoid spurious results. Specifically, let $\widehat{\boldsymbol{n}}(\boldsymbol{r})$ and $\widehat{\boldsymbol{n}}^{\prime}\left(\boldsymbol{r}^{\prime}\right)$ be neighboring local directors stored on a regularly meshed grid where $\boldsymbol{r}^{\prime} \equiv \boldsymbol{r} \pm \delta \boldsymbol{r} \cdot \widehat{\boldsymbol{e}}_{\alpha}$ and $\delta \boldsymbol{r}$ is the mesh width $(\alpha=x, y, z)$. For any one of the six local directors $\widehat{\boldsymbol{n}}^{\prime}\left(\boldsymbol{r}^{\prime}\right)$ that are nearest neighbors of $\widehat{\boldsymbol{n}}(\boldsymbol{r})$ on the grid, $\widehat{\boldsymbol{n}}^{\prime}\left(\boldsymbol{r}^{\prime}\right)$ is replaced by $-\widehat{\boldsymbol{n}}^{\prime}\left(\boldsymbol{r}^{\prime}\right)$ if the condition $\widehat{\boldsymbol{n}}(\boldsymbol{r}) \cdot \widehat{\boldsymbol{n}}^{\prime}\left(\boldsymbol{r}^{\prime}\right)<0$ is satisfied. Notice that this deliberate change in sign prior to the numerical differentiation of $\widehat{\boldsymbol{n}}(\boldsymbol{r})$ does not affect the final result for $\mathcal{F}(x, z)$ which depends quadratically on the local derivatives of $\widehat{\boldsymbol{n}}(\boldsymbol{r})$. All local quantities are analyzed in terms of two-dimensional histograms based upon a mesh width of $\delta x=\delta z=0.1$.

\section{RESULTS}

We begin the discussion of our results in Fig. 5 by illustrating the impact of the range of the fluid-substrate potential
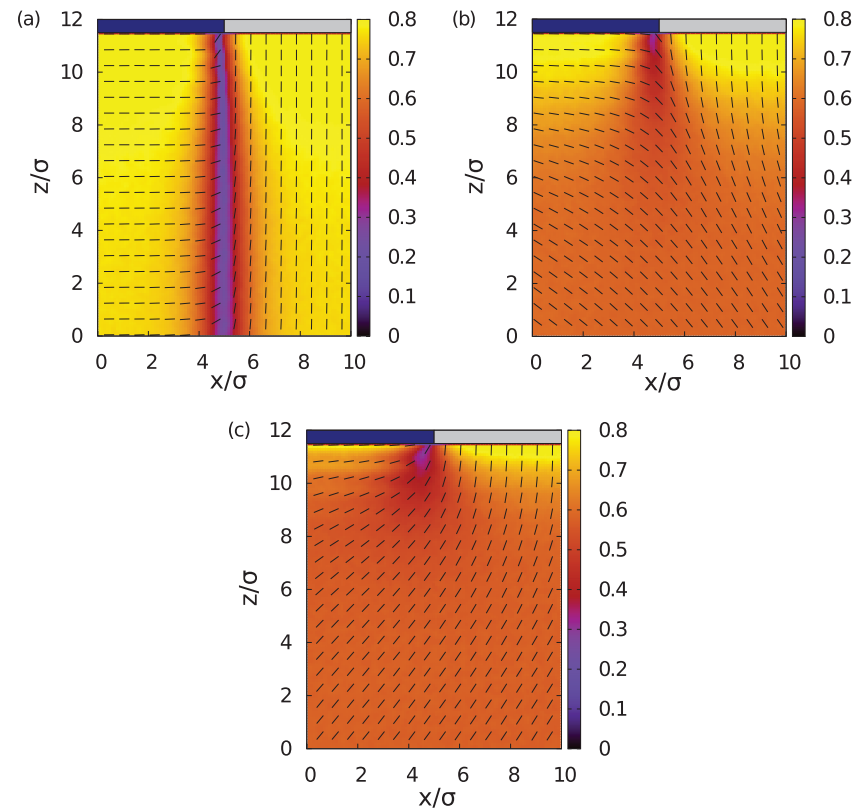

FIG. 5. Plots of the local nematic order parameter $\lambda(x, z)$ in the $x-z$ plane (see attached color bar) for $d_{\mathrm{w}}=10$ and hd anchoring (see Table I). Dashed lines refer to the local nematic director $\widehat{\boldsymbol{n}}(x, z)$ projected onto the $x-z$ plane. (a) $\kappa \sigma=0.00$, (b) $\kappa \sigma=0.45$, and (c) $\kappa \sigma=2.00$. For symmetry reasons data are plotted only in the quadrant $x \geq 0$ and $z \geq 0$ (see area shaded in red in Fig. 1). Gray and blue bars at the top of the plots represent parallel (p or d) and perpendicularly $(\mathrm{h})$ anchoring stripes, respectively. For convenience these bars have been placed in front of the substrates (i.e., inside the system) where, however, no mesogens are located on account of the strongly diverging fluidsubstrate interaction potential. through plots of both the local order parameter $\lambda(x, z)$ and the local nematic director field $\widehat{\boldsymbol{n}}(x, z)$. Plots in Fig. 5(a) have been generated for the completely unshielded, longest-range Yukawa attraction at $\kappa \sigma=0.00$. One notices two regions in which $\lambda(x, z)$ is relatively large and where $\widehat{\boldsymbol{n}}(x, z)$ reflects a homogeneous, parallel, or perpendicular molecular orientation with respect to the $x$-axis. Both regions are separated by a thin film of low local nematic order in which the molecular orientation changes from parallel for $|x|<d_{\mathrm{w}} / 2$ to perpendicular for $|x|>d_{\mathrm{w}} / 2$.

For $\kappa \sigma=0.45$ the fluid-substrate potential is moderately shielded. Plots of $\lambda(x, z)$ and $\widehat{\boldsymbol{n}}(x, z)$ in Fig. 5(b) indicate that the regions of nearly perfect parallel and perpendicular molecular orientation are now restricted to regions in the vicinity of those parts of the substrate at which molecules are directionally and homeotropically anchored, respectively. That is, the range over which the chemical pattern of the substrate is imprinted onto the adjacent nematic liquid crystal is limited by the range of the fluid-substrate interactions as expected. However, sufficiently far away from the substrate surfaces (i.e., for $z \lesssim 6$ ) Fig. 5(b) reveals that nematic order remains relatively large with $\lambda(x, z) \approx 0.5-0.6$ irrespective of position in the $x$ $z$ plane. Notice that this value agrees with the bulk value of the nematic order parameter 0.58 under the present thermodynamic conditions as one would anticipate (see Sec. III). The corresponding local director field shows that molecules have a small preference to align in a slightly more parallel fashion for $|x|<d_{\mathrm{w}} / 2$ whereas a more perpendicular orientation is observed for $|x|>d_{\mathrm{w}} / 2$. As one approaches the mid-plane at $|z| \simeq 0$ the director field becomes independent of position along the $x$-axis such that the molecular orientation forms a nonzero angle with the substrate normal. This angle is a fingerprint of orientational frustration which arises because the molecules try to compromise between the parallel orientation imposed by the directional anchoring and the perpendicular one enforced by the outer, homeotropically anchoring parts of the substrates. ${ }^{20}$

If the range of the fluid-substrate potential is even shorter plots in Fig. 5(c) for $\kappa \sigma=2.00$ show that the liquid crystal becomes even more homogeneous with respect to the local nematic order and the orientation of the local director field. Comparing plots in Figs. 5(b) and 5(c) also reveals that the orientation reflected by $\widehat{\boldsymbol{n}}(x, z)$ is different. This difference is caused by the fact that both orientations of the director field are energetically equivalent and hence they form with equal probability. We have checked this by a number of runs where we have driven an isotropic fluid with randomly distributed molecular orientations to a nematic state and found that both orientations of the director field arise more or less randomly with roughly the same probability of occurrence.

If one varies $d_{\mathrm{w}}$ while holding $\kappa \sigma=0.45$ fixed the larger one of the differently anchoring substrates will dominate the local nematic director field. This can be seen from plots in Figs. 6(a) and 6(b) for $d_{\mathrm{w}}=4$ and $d_{\mathrm{w}}=16$, where $\widehat{\boldsymbol{n}}(x, z)$ is mostly perpendicular and parallel with the substrate, respectively. Whereas in Fig. 6(a) the region of parallel molecular orientation is encircled by a nearly isotropic transition region in which $\widehat{\boldsymbol{n}}(x, z)$ changes from planar to perpendicular orientation over a relatively short distance a similar effect is 

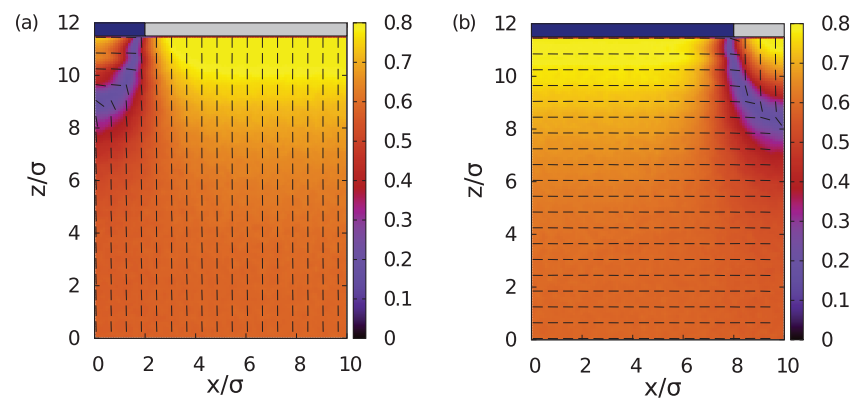

FIG. 6. As Fig. 5(b), but for (a) $d_{\mathrm{w}}=4$ and (b) $d_{\mathrm{w}}=16$.

observed for $d_{\mathrm{w}}=16$ in Fig. 6(b). Inside these channels (along the $y$-axis) encircled by the isotropic transition regime the local orientation imprinted by the adjacent chemical stripe of the substrate is preserved.

The deformations of the director field for the hd anchoring scenario are best discussed based on plots of the Frank free energy density $\mathcal{F}(x, z) / K$ in Fig. 7 [see Eq. (3.6)]. Let us begin the discussion with Fig. 7(a) where we present the Frank free-energy density for the topology of the director field shown in Fig. 5(b). Sufficiently far away from the junctions between neighboring chemical stripes at $|x| \approx 0$ and $|x| \approx s_{\mathrm{z}} / 2$ but close to the substrate for $|z| \gtrsim 10$ deformations of the local director vanish as indicated by $\mathcal{F}(x, z) / K \simeq 0$. However, as one approaches the junction $|x| \approx d_{\mathrm{w}} / 2$ the different anchoring directions cause a relatively strong distortion of the local director field which is largest in regions of lowest nematic order. At intermediate distances from the substrate, that is for $6 \lesssim|z| \lesssim 9$ the local nematic director field depends on $x$ over nearly the whole range $0 \leq|x|$ $\leq s_{\mathrm{X}} / 2$. These director distortions are also reflected by small values of $\mathcal{F}(x, z) / K$ which is characterized by a small but broad maximum around $|x| \approx d_{\mathrm{w}} / 2$. Since the director field becomes independent of $x$ as one approaches the mid-plane of the system the Frank free-energy density vanishes in that region.

However, plots in Fig. 6 indicate that globally the director field $\widehat{\boldsymbol{n}}(x, z)$ is of biaxial symmetry. The local orientation of the mesogens is mostly perpendicular and parallel with the substrate plane. Because of the homogeneity of the nematic director in these regions the elastic forces, which are caused by distortions of the local nematic director field [see
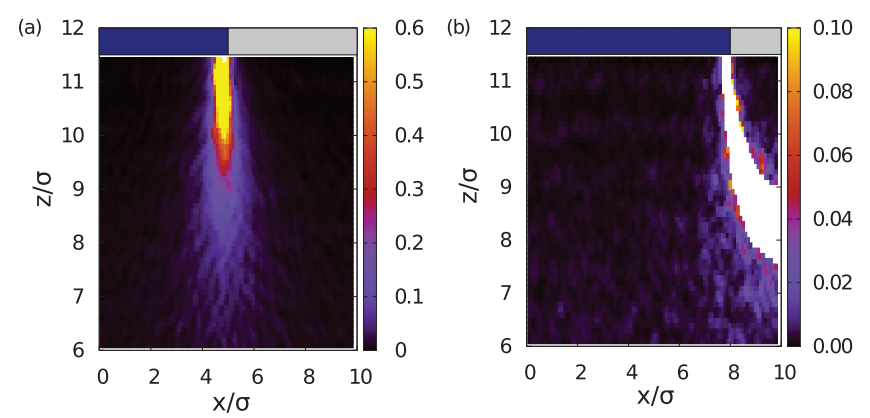

FIG. 7. Plots of the Frank free energy density $\mathcal{F}(\boldsymbol{r}) / K$ in the $x-z$ plane (see attached color bar) for $\kappa \sigma=0.45$ and hd anchoring. (a) $d_{\mathrm{w}}=10$ and (b) $d_{\mathrm{w}}=16$. Notice the difference in scale between parts (a) and (b) of the figure.
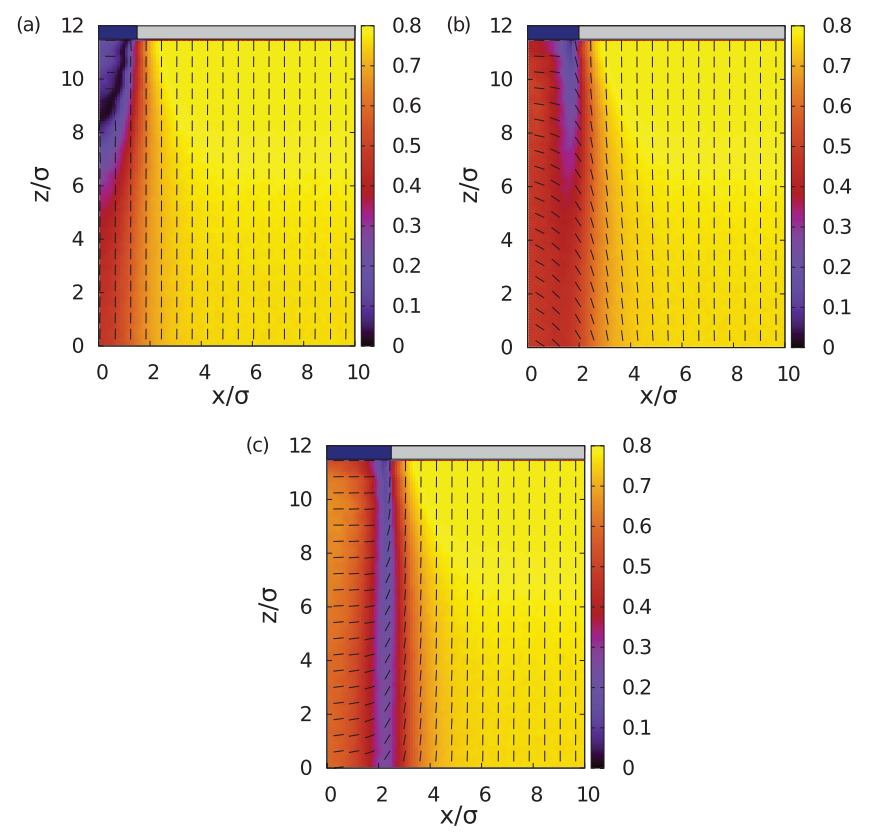

FIG. 8. As Fig. 5(a), but for hp anchoring, $\kappa \sigma=0.00$, (a) $d_{\mathrm{w}}=3$, (b) $d_{\mathrm{w}}=4$, and (c) $d_{\mathrm{w}}=5$.

Eq. (3.6)], are minimal and only differ considerably from zero close to the isotropic interface encircling the region in which the orientation of mesogens is controlled by the narrow chemical stripe on the substrate. The isotropic region is represented by the white area in Fig. 7 for which $\mathcal{F}(x, z) / K$ is undefined.

So far we have been considering combinations of monostable $\mathrm{h}$ and $\mathrm{d}$ anchoring scenarios at the chemically distinct stripes of the substrates. It is now interesting to compare the previously discussed results with those for degenrate planar and monostable homeotropic anchoring. We begin by displaying in Fig. 8 results that are obtained for an unshielded fluidsubstrate interaction (i.e., for $\kappa \sigma=0.00$ ) and various widths of the planar anchoring substrate part.

For the narrowest planar anchoring chemical stripe of width $d_{\mathrm{w}}=3$ plots in Fig. 8(a) show that on average a weak tendency towards parallel molecular alignment with the substrate prevails for $|x| \leq 1.5$ and $|z| \gtrsim 10$. However, $\lambda(x, z)$ remains relatively low in this region. On the contrary, $\lambda(x, z)$ attains values characteristic of the nematic phase for $|x| \geq 1.5$ irrespective of $z$. In this regime molecules exhibit an orientation perpendicular to the substrate plane.

To gain a somewhat deeper insight into local orientation effects we amend our analysis of $\lambda(x, z)$ in Fig. 8(a) by that of $P_{\mathrm{x}}(x, z)$ and $P_{\mathrm{z}}(x, z)$ in Fig. 9. The plot in Fig. 9(a) reveals a relatively large positive value of $P_{\mathrm{z}}(x, z)$ almost everywhere in the liquid crystal except for the tiny region controlled by the parallel anchoring chemical strips. However, in this region where nematic order is low [cf., Fig. 10(a)] a preferential parallel alignment of the mesogens with the substrate plane prevails as a comparison between plots in Figs. 9(b) and 9(c) indicates. Notice in particular that $P_{\mathrm{x}}(x, z)>P_{\mathrm{z}}(x, z)$ and that $P_{\mathrm{x}}(x, z)>0$ in the region controlled by the parallel anchoring chemical stripe. These features reflect a tendency of the mesogens to assume a weak parallel alignment with the substrate plane. This notion is supported by the plot of $P_{\mathrm{z}}(x, z)$ 

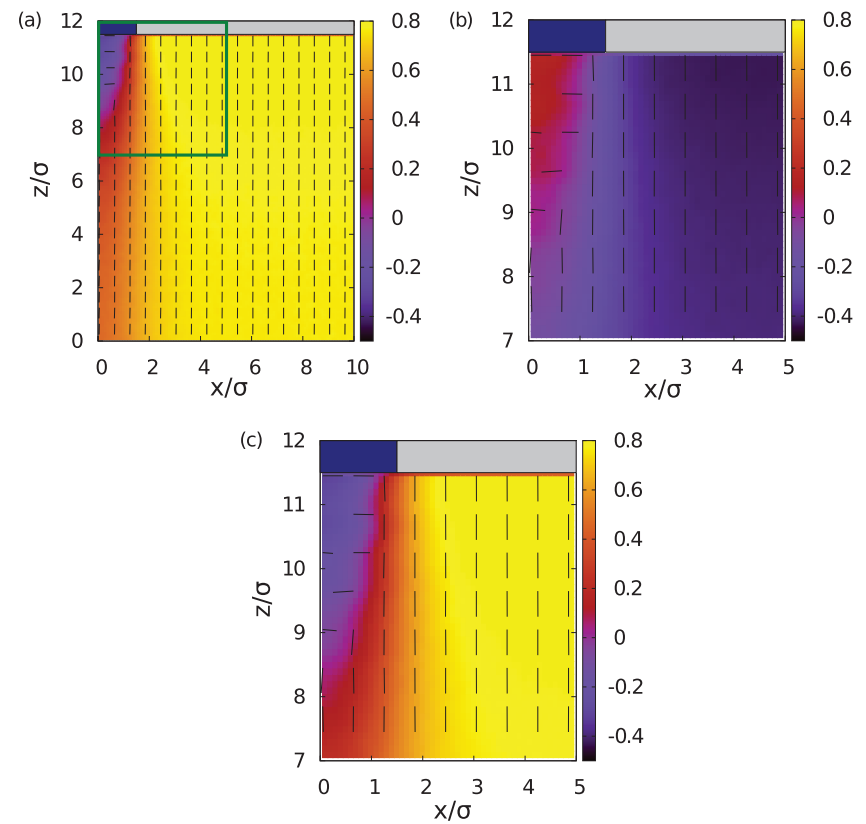

FIG. 9. Plots of the order parameter $P_{\alpha}$ in the $x-z$ plane (see attached color bar) for $\kappa \sigma=0.00, d_{\mathrm{w}}=3$, and hp anchoring. (a) $P_{z}$, (b) $P_{x}$, and (c) $P_{z}$. (b) and (c) Enhancements of data obtained for the region surrounded by green lines in (a) of the figure.

in Fig. 9(c) which shows that in the region where $P_{\mathrm{x}}(x, z)$ $>0, P_{\mathrm{z}}(x, z) \simeq-0.4$ indicating an almost complete absence of homeotropic alignment. We have also analyzed $P_{\mathrm{y}}(x, z)$ which turns out to satisfy the relation $P_{\mathrm{x}}(x, z) \simeq P_{\mathrm{y}}(x, z)$ which indicates that the local director lies somewhere on the unit circle in the $x-y$ plane.

For a slightly wider central stripe plots in Fig. 8(b) show that the imprinted parallel and perpendicular orientation of molecules in the vicinity of the substrate (i.e., for $z \geq 10$ ) are separated by a region of relatively low nematic order that ex-
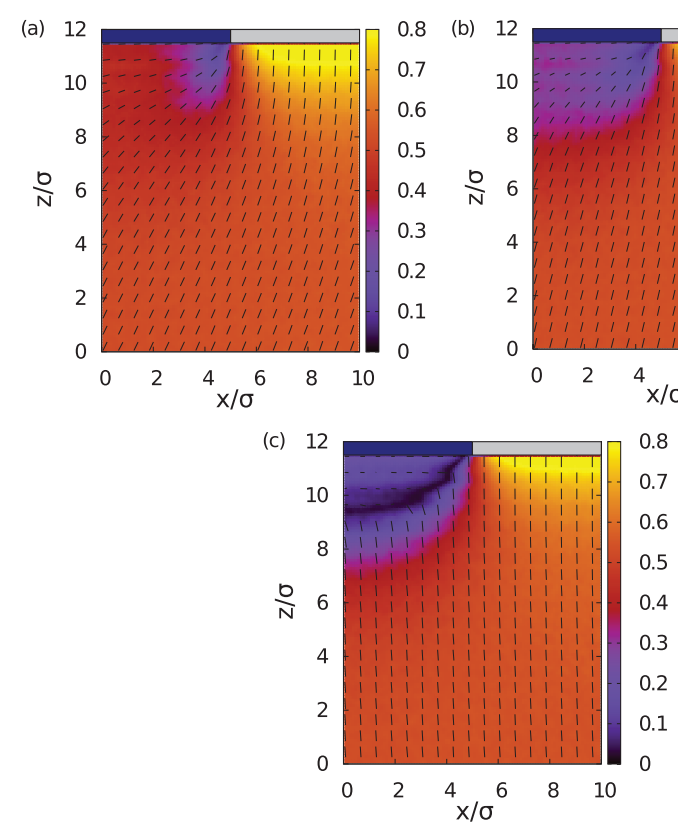

FIG. 10. As Fig. 8(c), but for different ranges of the fluid-substrate attraction; (a) $\kappa \sigma=1.00$, (b) $\kappa \sigma=1.40$, and (c) $\kappa \sigma=2.00$. tends into the more bulk-like regions of the liquid crystal $z$ $\geq 6$. Over the homeotropically anchoring portion of the substrate $(|x| \geq 2)$ the perpendicular orientation of the molecules imprinted by the substrate extends all the way down to the midplane located at $z=0$. On the contrary, molecular orientation over the planar anchoring stripe $(|x| \leq 2)$ changes from parallel in the immediate vicinity of the substrate to a tilted orientation with respect to the substrate plane as one approaches the midplane of the system which reflects again orientational frustration of the molecules. ${ }^{20}$ This region of orientational frustration changes for a slightly wider central stripe to a well-defined thin layer in Fig. 8(c) of low nematic order and frustrated molecular orientation if $d_{\mathrm{w}}=5$ is considered. This thin layer separates two regions of orthogonal orientations where for $|x| \lesssim 2.5$ mesogens appear to be only marginally tilted. Hence, the global orientation of the liquid crystal again exhibits biaxial symmetry. The parallel alignment becomes nearly perfect if a substrate with chemical stripes of equal widths $d_{\mathrm{w}}=10$ is considered [see Fig. 5(a)] which shows that the anchoring at the substrate may be imprinted onto an adjacent bulk-like nematic phase.

However, comparing $\lambda(x, z)$ in the regime of parallel alignment with the one in the region where the mesogens exhibit homeotropic alignment it turns out that $\lambda(x, z)$ is lower in the former. This is because degenerate planar anchoring is weaker than monostable homeotropic anchoring. The different degrees of local nematic order may be rationalized by entropic arguments. In the formation of ordered phases the mesogens lose rotational configurational entropy. This loss is larger in the case of monostable homeotropic anchoring, where the mesogens have to align with a single easy axis, compared with degenerate planar anchoring where an infinite number of easy axes exists on the unit circle in the $x-y$ plane. Hence, the stability of the ordered phase is expected to be larger for the monostable anchored case which should result in a larger degree of nematic order. From this line of arguments it follows that in cases where the substrate is decorated with chemical stripes that both anchor molecules in a monostable way the degree of local nematic order should be roughly the same. The plot in Fig. 5(a) confirms this notion.

As expected the strong imprinting of the specific anchoring by the substrate diminishes as one reduces the range of fluid-substrate attraction $\kappa$ while maintaining the relative widths of the different anchoring stripes. For $\kappa \sigma=1.00$ plots in Fig. 10(a) show that a homogeneous region of frustrated molecular orientation develops towards the midplane at $z=0$. The specific anchoring of the stripes can be imprinted onto the liquid crystal only over a relatively short range of distances from the substrate. In the immediate vicinity of the substrates and at the junction between planar and homeotropically anchoring stripes a region of low nematic order exists. This region grows towards the center of the planar anchoring stripe as $\kappa$ increases. A comparison between the sequence of plots presented in Figs. 10(a)-10(c) also shows that the frustrated molecular orientation near the midplane visible in Fig. 10(a) changes to a nearly perfect perpendicular one in the entire system outside the isotropic region near the planar anchoring 
stripe, again indicating the greater strength of monostable anchoring scenarios.

\section{SUMMARY AND CONCLUSIONS}

By means of MC simulations in the grand canonical ensemble we investigate the impact of a chemically patterned substrate on the local order and director field of a nematic liquid crystal. The pattern consists of alternating stripes of variable width. The stripes anchor molecules in different ways. We consider combinations of homeotropically and directionally anchoring stripes both of which belong to the class of monostable anchoring scenarios. ${ }^{25}$ In addition, the effect of a combination of monostable homeotropic anchoring and of degenerate planar anchoring is investigated.

The observed defect topologies depend crucially on the relative widths of the different stripes and on the range of the fluid-substrate attraction. The latter is modelled by a Yukawa potential which allows for a continuous variation of the attraction range via the shielding parameter $\kappa$. For the longest range attraction and equally wide stripes the molecular orientation imposed by monostable homeotropic anchoring and degenerate directional anchoring stripe is imprinted nearly perfectly onto the adjacent nematic liquid crystal. Regions of nearly perfect homogeneous homeotropic and parallel alignment of molecules are separated by a thin region of low nematic order in which the director field is distorted over a short distance of about one molecular diameter. If the range of fluid-substrate attraction is reduced the anchoring imposed by different parts of the substrate cannot be imprinted onto the liquid crystal but causes frustration of the molecular orientation. This frustration is signaled by a molecular orientation which may be viewed as a compromise between perfect parallel and homeotropic orientations.

If one instead maintains the longest range attraction but varies the width $d_{\mathrm{w}}$ of the planar anchoring stripe the director field is mostly dominated by the homeotropic anchoring imposed by the two outer stripes for small $d_{\mathrm{w}}$. As $d_{\mathrm{w}}$ increases the director field in that part of the system controlled by the planar anchoring stripe exhibits orientational frustration which eventually changes to a perfect planar orientation if a critical stripe width is reached. The planar orientation as dictated by the inner stripe is separated by a thin layer from the remainder of the liquid crystal where a homeotropic orientation prevails.

An interesting phenomenon is observed if the width of an outer monostable anchoring and the inner degenerate anchoring stripe are the same but the range of fluid-substrate attraction is gradually reduced. In this event a region of low nematic order develops over the planar anchoring substrate as $\kappa$ increases. The director field outside the isotropic regime is then dictated by the homeotropically anchoring substrate if the isotropic regime near the planar anchoring stripe is fully developed. Thus, the symmetry of the director field is uniaxial in regions of high nematic order. Another conclusion that can be drawn from our results is that the director field is biaxial in the vicinity of the substrates for stripes of equal width.
The bulk-like region sufficiently far removed from the substrate surfaces exhibits a local director field that is bent on account of orientational frustration. As $\kappa$ decreases eventually a fully developed state occurs in which the director field has biaxial symmetry even though our molecules are of uniaxial symmetry.

States of biaxial symmetry also arise for moderately long-range fluid-substrate attraction if both stripes are characterized by a monostable anchoring scenario. If, in this case, one of the stripes is much smaller (larger) than the other one a small region exists in which mesogens are almost perfectly aligned. This region is encircled by a transition zone in which the confined liquid crystal is isotropic. This zone then separates the small region from the remainder of the liquid crystal in which mesogens orient themselves in a direction orthogonal to the molecular orientation in the small region.

\section{ACKNOWLEDGMENTS}

We thank Jan Genzer (North Carolina State University) for discussions. Two of us (M.G. and M.S.) wish to thank K.E.G. for his hospitality during an extended research visit and a sabbatical spent in his group. We are grateful for financial support from the International Graduate Research Training Group 1524 "Self-assembled soft-matter nanostructures at interfaces." K.E.G. thanks the U.S. National Science Foundation for a grant (OISE-1065466) in support of this research. The authors are grateful to Vinothkumar Mohanakrishnan for help with the figures.

${ }^{1}$ P. Yeh, Optics of Liquid Crystal Displays (Wiley, New York, 2009).

${ }^{2}$ M. Humar, M. Ravnik, S. Pajk, and I. Muševič, Nat. Photonics 3, 595 (2009).

${ }^{3}$ O. Guzmán, N. L. Abbott, and J. J. de Pablo, J. Chem. Phys. 122, 184711 (2005).

${ }^{4}$ B. W. Lee and D. Sagong, Mol. Cryst. Liq. Cryst. 364, 519 (2001).

${ }^{5}$ V. K. Gupta and N. L. Abbott, Science 276, 1533 (1997).

${ }^{6}$ X. Li, X. M. Lu, Q. H. Lu, and C. A. Ohlin, Jpn. J. Appl. Phys. 245, L591 (2006)

${ }^{7}$ X. Jiang, H. Zeng, S. Gourdin, and P. T. Hammond, Langmuir 18, 2607 (2002)

${ }^{8}$ H. T. A. Wilderbeek, J.-P. Teunissen, C. W. M. Bastiaansen, and D. J. Broer, Adv. Mater. 15, 985 (2003).

${ }^{9}$ I. H. Bechtold and E. A. Oliveira, Liq. Cryst. 32, 343 (2007).

${ }^{10}$ T. J. Atherton, J. R. Sambles, J. P. Bramble, J. R. Henderson, and S. D. Evans, Liq. Cryst. 36, 353 (2009).

${ }^{11}$ J.-H. Kim, M. Yoneya, and J. Yamamoto, Appl. Phys. Lett. 78, 3055 (2001).

${ }^{12}$ S. Kondrat and A. Poniewierski, Phys. Rev. E 64, 031709 (2001).

${ }^{13}$ A. Poniewierski and S. Kondrat, J. Mol. Liq. 112, 61 (2004).

${ }^{14}$ L. Harnau, S. Kondrat, and A. Poniewierski, Phys. Rev. E 72, 011701 (2005).

${ }^{15}$ S. Kondrat, A. Poniewierski, and L. Harnau, Eur. Phys. J. E 10, 163 (2003).

${ }^{16}$ F. K. P. Haddadan and S. Dietrich, Phys. Rev. E 73, 051708 (2006).

${ }^{17}$ T. J. Atherton and J. R. Sambles, Mol. Cryst. Liq. Cryst. 475, 3 (2007).

${ }^{18}$ T. J. Atherton, Liq. Cryst. 10, 1225 (2010).

${ }^{19}$ M. Greschek, M. Melle, and M. Schoen, Soft Matter 6, 1898 (2010).

${ }^{20}$ M. Greschek and M. Schoen, Soft Matter 6, 4931 (2010).

${ }^{21}$ M. Greschek and M. Schoen, Phys. Rev. E 83, 011704 (2011).

${ }^{22}$ M. Greschek and M. Schoen, J. Chem. Phys. 135, 204702 (2011).

${ }^{23}$ S. Hess and B. Su, Z. Naturforsch. 54a, 559 (1999).

${ }^{24}$ J. S. Rowlinson, Physica A 156, 15 (1989).

${ }^{25}$ B. Jerôme, Rep. Prog. Phys. 54, 391 (1991).

${ }^{26}$ T. Gruhn and M. Schoen, Phys. Rev. E 55, 2861 (1997).

${ }^{27}$ D. Frenkel and B. Smit, Understanding Molecular Simulation (Academic, San Diego, 2002), Chap. 5.6. 
${ }^{28}$ M. P. Allen and D. J. Tildesley, Computer Simulation of Liquids (Clarendon, Oxford, 1987), Chap. 5.3.

${ }^{29}$ W. H. Press, S. A. Teukolsky, W. T. Vetterling, and B. P. Flannery, Numerical Recipes in FORTRAN (Cambridge University Press, Cambridge, 1989), Chap. 11.1.

${ }^{30}$ D. Andrienko, G. Germano, and M. P. Allen, Phys. Rev. E 63, 041701 (2001).

${ }^{31}$ R. Eppenga and D. Frenkel, Mol. Phys. 52, 1303 (1984).
${ }^{32}$ C. Zannoni, The Physics of Liquid Crystals (Academic, London, 1979).

${ }^{33}$ H. Weber, W. Paul, and K. Binder, Phys. Rev. E 59, 2168 (1999).

${ }^{34}$ A. Richter and T. Gruhn, J. Chem. Phys. 125, 064908 (2006).

${ }^{35}$ M. Melle, S. Schlotthauer, M. G. Mazza, S. H. L. Klapp, and M. Schoen, J. Chem. Phys. 136, 194703 (2012).

${ }^{36}$ P. G. de Gennes and J. Prost, The Physics of Liquid Crystals (Oxford University Press, Oxford, 1995), Chap. 3.1. 